\title{
La naturaleza de los bienes y servicios para evaluación social de la vida
}

Una evaluación de impacto debe abarcar los siguientes aspectos: describirla acción propuesta, así como otras alternativas; predecir la naturaleza y magnitud de los 'efectos ambientales'; predecir los aspectos humanos; interpretar los resultados; y prevenir los efectos ambientales. Además, hay que disponer de una metodología para las fases de comunicación (información al público y al ejecutivo) y, de acuerdo con el caso, para los procedimientos de inspección durante la fase de construcción y de operación del proyecto o la acciones de que se trate.

Palabras clave: Bienes y servicios; Evaluación; Sociedad.

\section{A natureza dos bens e serviços para avaliação social da vida}

Uma avaliação de impacto deve abranger os seguintes aspectos: descrever a ação proposta, bem como alternativas; prever a natureza e magnitude dos 'efeito ambientais'; prever aspectos humanos, interpretar resultados; e prevenir os efeitos ambientais. Além disso, deve estar disponível uma metodologia para as fases de comunicação (informação ao público e ao executivo) e, conforme o caso, para os procedimentos de fiscalização durante a fase de construção e operação do empreendimento ou ação em causa.

Palavras-chave: Bens e serviços; Avaliação; Sociedade.

Topic: Biotecnologia

Reviewed anonymously in the process of blind peer
Received: $12 / 08 / 2020$

Approved: 15/12/2020
Cleber Vinicius Vitorio da Silva (iD Universidade Federal Rural do Rio de Janeiro, Brasil http://lattes.cnpq.br/4275890458575782 http://orcid.org/0000-0001-8337-9615 clebervitorio88@gmail.com

Eliana Beatriz Nunes Rondon Lima (iD) Universidade Federal de Mato Grosso, Brasil http://lattes.cnpq.br/9641750996845089 http://orcid.org/0000-0002-0378-9778 ebnrlima@gmail.com

Elenice Rachid da Silva Lenz (iD)

Universidade do Estado do Rio de Janeiro, Brasil http://lattes.cnpq.br/4557023865361858 http://orcid.org/0000-0002-1440-4271 erachid@pet.coppe.ufri.br

\section{Evandro Lima (ib)}

Universidade Federal Rural do Rio de Janeiro, Brasil

http://lattes.cnpq.br/7057930279111732

http://orcid.org/0000-0003-4213-9652

evandrolimaabpg@uol.com.br

Gustavo Aveiro Lins (iD

Universidade Federal Rural do Rio de Janeiro, Brasil http://lattes.cnpq.br/5173989372426437

http://orcid.org/0000-0002-0244-6925

gustavoaveiro@gmail.com

\section{Josimar Ribeiro de Almeida (iD)}

Universidade do Estado do Rio de Janeiro, Brasil

http://lattes.cnpq.br/3215586187698472

http://orcid.org/0000-0001-5993-0665

almeida@poli.ufri.br
Lais Alencar de Aguiar (iD)

Comissão Nacional de Energia Nuclear, Brasil

http://lattes.cnpq.br/5785500333245448

http://orcid.org/0000-0002-1551-4085

aguiar.lais@gmail.com

\section{Referencing this:}

SILVA, C. V. V.; LIMA, E. B. N. R.; LENZ, E. R. S.; LIMA, E.; LINS, G. A.; ALMEIDA, J. R.; AGUIAR, L. A.. La naturaleza de los bienes y servicios para evaluación social de la vida. Naturae, v.2, n.2, p.30-38, 2020. DOI: http://doi.org/10.6008/CBPC2674-6441.2020.002.0005 


\section{INTRODUCCIÓN}

El interés masivo por los temas ambientales surgió al final de los años sesenta. Fue entonces cuando se empezaron a percibir con nitidez los problemas de deterioro del medio ambiente, que en principio se centraron especialmente en la contaminación. El concepto medio ambiente tuvo en su época dos acepciones bastante claras, según lo que se aplicara en los países industrializados o en los países en vías de desarrollo. En los primeros, la temática ambiental se concentraba casi exclusivamente en los aspectos de contaminación, en cuyo caso resultaba correcto considerar que los problemas ambientales tenían un carácter tecnológico. En cambio, en los países en vías de desarrollo el concepto era mucho más amplio y de carácter socioeconómico y político, más que tecnológico, puesto que se consideraban problemas ambientales prioritarios, precisamente los derivados del subdesarrollo: problemas sanitarios, condiciones de los asentamientos humanos, falta de viviendas y escuelas, deficiencias de nutrición, de destrucción de bosques y pérdidas de suelos, destrucción o mala explotación de recursos naturales $u$ otros. A estos problemas del subdesarrollo hay que añadir los que pueden generarse de un desarrollo que no considere en sus proyectos la variable ambiental. Hoy en día, en todos los países, el concepto de medio ambiente tiene un sentido único y generalizado, cada vez más amplio, hasta el punto de que conceptos tan complejos y extensos como los de la calidad de vida y asentamientos humanos se integran en su temática. Persiste sin embargo la idea de que los problemas ambientales en los países industrializados derivan en su mayor parte de procesos de desarrollo y, muy especialmente, de las grandes concentraciones humanas y/o industriales (la contaminación, la deshumanización de la vida en las grandes ciudades, la creciente conflictividad social de las metrópolis, el consumo exagerado de recursos naturales y de energía, los problemas urbanísticos etc.), en cuanto que en los países en vías de desarrollo los problemas ambientales se deben, principalmente, al bajo índice de crecimiento. Las evaluaciones de impacto ambiental nacieron en los Estados Unidos, como consecuencia de la Ley Nacional de Política Ambiental de 1 de enero de 1970. Es en ese país donde se han hecho más trabajos de este tipo y, por consiguiente, donde se han desarrollado más metodologías para los mismos. Hasta la fecha se ha preparado muchas, pero ninguna de ellas tiene una dimensión o un carácter universal. Así pues, aunque existe gran cantidad de modelos, son pocos los que están sistematizados. La primera evaluación ambiental en Brasil fue realizada en 1972, una exigencia del Banco Mundial para el financiamiento de una represa y de una hidroeléctrica en Sobradinho, Bahia. Otros proyectos como el de la hidroeléctrica de Tucuruí, en Paraná, y el del terminal del puerto ferroviario Ponta de Madeira, en Maranhão, punto de exportación del minero extraído por la CVRD, en Serra dos Carajás fueron subordinados a la AIA en la década de 70 e inicio de la década de 80 (MONTANDON et al., 1989).

\section{REPORTE}

Se dice que hay un impacto ambiental cuando una acción o actividad produce una alteración en el medio o en alguno de los componentes del medio. Por lo tanto, la variable fundamental en estos estudios es la cuantificación de la alteración. En los estudios de impacto ambiental se trata de evaluar las consecuencias 
de una acción, para ver la calidad del ambiente que habría con o sin dicha acción. Tales evaluaciones deben realizarse en la fase previa al proyecto, antes que éste se realice, con objeto de: a) efectuar una mejor planificación y formulación de propuestas, desde el punto de vista ambiental, y b) considerar adecuadamente los factores ambientales, por parte de las autoridades, cuando aprueben una propuesta o determinen una alternativa. Se aplica el concepto de evaluación del impacto ambiental a un estudio encaminado a identificar e interpretar, así como a prevenir, las consecuencias los efectos que acciones o proyectos determinados pueden causar a la salud y al bienestar humanos y al entorno, o sea, en los ecosistemas en que el hombre vive y de los que depende. La dificultad para demostrar la complejidad de la dinámica ambiental en una única definición hace que, en general, todas las definiciones adquieran un carácter reduccionista y estático. Esto constituye, de cierto modo, el gran problema de las conceptualizaciones. Otras dificultades encontradas en la definición y, especialmente, en la identificación de un impacto ambiental consisten en la propia delimitación del impacto, una vez que el mismo se propaga espacial y temporalmente a través de una compleja red de interrelaciones y, también, en las deficiencias instrumentales y metodológicas para prever las respuestas de los ecosistemas a las actividades antrópicas. Esta cuestión es todavía más crítica cuando se trata de la dimensión social. Los estudios deben considerar las alternativas de la acción y del proyecto. Éstos también presuponen la participación del público representando, no solamente un instrumento de decisión, sino también un instrumento de conocimiento al servicio de la decisión. Existen innumerables definiciones en la literatura especializada del proceso de evaluación de impactos ambientales. La mayoría es de origen académica, enfatizando aspectos técnicos. Otras dan énfasis a los componentes políticos de gestión ambiental. Existen también las definiciones legales, como la instituida en el NEPA. Para ser útil, la evaluación debe ser comunicada en términos comprensibles para la comunidad y para los responsables por la toma de decisión. Los pros y contras deben ser identificados en base a criterios relevantes para los países afectados" (SILVA et al., 2014); - “... es una actividad destinada a identificar y prever el impacto sobre el ambiente biogeofísico y sobre la salud y el bienestar de los seres humanos, resultante de propuestas legislativas, políticas, programas y proyectos y de sus procesos operacionales y de interpretar y comunicar las informaciones sobre estos impactos" (ALMEIDA, 2011);- El término "estimativa ambiental" describe la técnica y el proceso por el cual se colecta información acerca de los efectos ambientales de un proyecto, tanto los producidos por el que lo desarrolla como los generados por otras fuentes. Debe considerarse, inclusive, si el desarrollo amerita seguir enfrente o no, por medio de juicio formado, gracias a la autoridad profesional (ALMEIDA, 2011). - “...instrumento de política ambiental, formado por un conjunto de procedimientos, capaz de asegurar, desde el inicio del proceso, que se haga un examen sistemático de los impactos ambientales de una acción propuesta(proyecto, programa, plan o política) y de sus alternativas, y que los resultados sean presentados de forma adecuada al público y a los responsables por la toma de decisión, y por ellos considerados. Además de eso, los procedimientos deben garantizar la adopción de las medidas de protección del medio ambiente determinadas, en el caso de decisión sobre la implantación del proyecto (ALMEIDA et al., 2018)). - Una estimativa del impacto de una actividad planeada en el ambiente (SILVA et al., 2012)). En suma, la evaluación de impactos ambientales es "un instrumento de política 
ambiental, formado por un conjunto de procedimientos, capaz de asegurar, desde el inicio del proceso, que se haga un examen sistemático de los mismos en una acción propuesta (proyecto, programa, plan o política) y de sus alternativas. Además, que los resultados sean presentados de forma adecuada al público y a los responsables por la toma de decisiones, debidamente considerados por éstos" (ALMEIDA et al., 2018). Según este autor, las definiciones identifican importantes y distintos componentes. Uno de ellos es el que engloba un conjunto de procedimientos para identificar, evaluar y prevenir efectos adversos y que debe estar relacionado con conocimiento científico sobre el ambiente, la acción y sus interrelaciones. El otro componente es el proceso de toma de decisión, en el cual la evaluación de impactos de una acción puede tener un importante papel que está íntimamente relacionado con reglas administrativas y voluntad política. En la literatura de habla inglesa se adoptan términos, como 'Environmental Impact Assessment' (EIA) para designar estudios que engloban conjuntamente aspectos sociales y ecológicos, y 'Ecological Impact Assessment' y 'Social Impact Assessment' para los que tratan de aspectos ecológicos y sociales, respectivamente. Un término que engloba mejor mejores los estudios es el 'Integrated Impact Assessment', que se refiere al estudio del conjunto de consecuencias sociales y ecológicas según un enfoque holístico, que ponga en evidencia los efectos cumulativos resultantes de sus interacciones requiriendo para su elaboración un conjunto de disciplinas diferentes, pero integradas. En los Estados Unidos, por ejemplo, las evaluaciones de impactos ambientales se reflejan en un documento denominado 'Environmental Impact Statement' (EIS). La agencia responsable por la evaluación emite un 'Notice of Intent' (NOI), que es enviado a otras agencias federales, estaduales y locales, a los impulsores del proyecto y a los grupos e individuos interesados. A continuación, con el intento de discutir el desarrollo del EIS, es realizado un 'scoping meeting' en el cual se ha observado una creciente participación de representantes de la comunidad. Mientras transcurre el proceso de evaluación, el público tiene acceso al 'draft' del EIS para comentarios y solicitud de esclarecimientos que pueden ser incorporados o anexados a la versión final del documento. Ante la ausencia de palabras correspondientes, en lengua española utilizamos 'Evaluación' tanto para designar 'Assessment' como 'Evaluation'. Westman, sin embargo, se define 'Assessment' como análisis y 'Evaluation', como evaluación de impacto. El análisis consiste en una tarea objetiva de identificación de acciones, medición de las condiciones de base y previsión de los probables cambios de las condiciones resultantes de aquellas acciones. La evaluación se constituye en una tarea objetiva o normativa que depende de la aplicación de valores humanos, ya que incluye determinar la significación de los efectos de los impactos ambientales. La concepción de Westman para la evaluación demuestra, en parte, tendencias del desarrollo de un proceso en el aspecto ecológico, no considerando, por tanto, los aspectos sociales. Cox \& Tait también dividieron la Estimativa de Riesgo en dos componentes, en la Análisis de Riesgo y la Evaluación de Riesgo. Durante la referida evaluación se hacen anotaciones sobre el tipo de trabajo a realizarse encada área, la planta y los equipos a ser utilizados, así como un inventario de sustancias peligrosas para la salud, las personas responsables y otros detalles relevantes. La segunda etapa en la estimativa de riesgos se refiere a la identificación de peligros. La institución de Ingenieros Químicos definir la palabra peligro como" una situación física con potencial para causar daños a la humanidad, a las propiedades, al ambiente o a la combinación de 
estas. La tercera etapa de la estimativa de riesgos está relacionada al informe de La Sociedad Real que define el riesgo como 'la combinación de la frecuencia o probabilidad de los acontecimientos de un peligro definido, y la magnitud de la consecuencia de lo sucedido'. De la misma forma, la institución de Ingenieros Químicos define el riesgo como 'la posibilidad de que un evento específico e indeseable ocurra dentro de un período específico o en circunstancias específicas. Podría también definirse como frecuencia (número de eventos específicos que ocurren en una unidad de tiempo) o probabilidad (probabilidad de un evento específico suceder a uno anterior). La evaluación de riesgos sería simplificada si pudiéramos desarrollar una medida, expresada en términos de los dos factores usados para definirlos matemáticamente, que suministraría una representación útil del riesgo observado. Ha habido algunas discusiones al respecto, pero experiencias prácticas indican que el producto de los dos factores provee una base adecuada para saber, por lo menos, donde los problemas comunes se presentan. El término 'público', normalmente utilizado en la documentación y literatura relativa a la evaluación de impactos ambientales puede estar sujeto a diferentes interpretaciones. Tanto puede hacer referencia a 'lo popular', como 'a un conjunto de personas que asisten a un espectáculo, a una reunión...'. Lo que observamos en el desarrollo histórico de las evaluaciones, sin embargo, es que prevalece la última definición. En este sentido, el público ha sido incorporado al final del proceso tan sólo como espectador y receptor de informaciones. Otros aspectos interesantes de la concepción de Westman residen en la inclusión en la fase de definición de objetivos y de monitoreos, fases que él denomina de pre y pós impacto, respectivamente. La primera induce a la ampliación y al mejor aprovechamiento de la discusión de los objetivos del estudio. La segunda, propicia una realimentación para la evaluación que opera, frecuentemente, con un elevado grado de incertidumbre.

\section{DISCUSIÓN}

Es importante resaltar que la investigación guiada por el método dialéctico revela la historicidad del fenómeno y sus relaciones, a un nivel más amplio, sitúan el problema dentro de un contexto complejo, al mismo tiempo, estableciendo y señalando las posibles contradicciones entre los fenómenos investigados. La investigación cualitativa se basa en la inseparabilidad de los fenómenos y su contexto, ya que las opiniones, percepciones y significados se entenderán con mayor profundidad desde el contexto. La validez se referiría a la similitud entre el concepto y sus medidas, el grado en que una medida representa precisamente lo que se espera. La garantía de validez comenzaría con una comprensión directa de lo que se debe medir, por lo tanto, se trata de formulación de la investigación. El método propuesto se dividió en tres fases, que se subdividieron en etapas para la realización del relevamiento bibliográfico. En la primera fase de la investigación (preguntas de investigación), se verifican las principales decisiones y definiciones sobre la investigación. Las preguntas de investigación se destacan y sirven como impulso para el inicio de la revisión. Se define el tema para definir la encuesta bibliográfica y el período disponible. Otras decisiones se toman simultáneamente, como definir palabras clave, para que actúen como identificadores. Paralelamente, resumen los principales temas sobre el tema a investigar; así como combinaciones de palabras clave. Estos, a su vez, se pueden realizar mediante operadores booleanos y en diferentes bases de datos como CAPES 
Journal Portal, SCIELO Database, Publish or Perish, EBSCO, entre otras. En el presente caso, las bases de datos fueron Web of Science (o ISI), Plataforma SUSTENERE, Scielo y Scopus. Estas bases de datos son de fácil acceso, lo que permite, a través de sus herramientas, realizar una cuidadosa investigación, abarcando un amplio número de revistas. La segunda fase (selección de artículos) inicia la encuesta y selección de artículos, utilizando los criterios de exclusión. Las palabras clave de exclusión se utilizan para realizar una clasificación más cuidadosa de los artículos. También delimitando el período. En el siguiente paso, se produce el primer filtro de los artículos, se realiza una selección de los títulos, identificando los no alineados. En el siguiente paso, se leen los resúmenes para excluir a aquellos que son impertinentes con el tema investigado. El siguiente paso contiene un análisis subjetivo por parte del investigador para identificar, en los artículos alineados con la temática, aquellos que tienen mayor relevancia académica. Un análisis bibliométrico indica la relevancia del autor/artículo para la composición de la referencia bibliográfica. La tercera fase corresponde a la clasificación de los artículos seleccionados. Se organizan muestras de artículos seleccionados. El objetivo es el orden, patrón que variará según las necesidades del investigador y lo priorice como foco de desarrollo temático. Destaca la importancia de un análisis exhaustivo de los artículos que componen la muestra. En la investigación cualitativa, el concepto de validez toma diferentes formas, ya que la discusión de las escalas de medición no se aplica a los métodos cualitativos, lo que requiere una comprensión de la validez desde otra perspectiva. Un atributo que se relaciona con la objetividad, la posibilidad de repetir el experimento, el hecho de que la investigación esté abierta a la verificación de otras personas y la capacidad de generalizar (ALMEIDA et al., 2013). La validez puede verse genéricamente como la correspondencia entre investigación y realidad (SILVA et al., 2012). Se refiere a verificar los resultados como verdaderos y confiables. Estaría relacionado con el hecho de que los resultados reflejan fielmente la situación analizada y son confiables, en el sentido de que no habría razón para dudar de ellos; es decir, la investigación es válida si la evidencia proporciona el soporte necesario para sus conclusiones (SOUZA et al., 2011). La intención no es generalizar, sino describir, analizar, buscar comprender.

\section{CONSIDERACIONES FINALES}

Muchos de los efectos ambientales resultan de la carencia de infraestructura y de fallas técnicas en la preparación del proyecto. Otras pérdidas resultan de la falta de información confiable sobre las consecuencias indirectas de los proyectos. Así no importa cuál sea la causa principal de la no incorporación de las externalidades en la formulación y ulterior evaluación de los proyectos; la colectividad tendrá que pagar un precio elevado para intentar remediar las decisiones erradas tomadas en el pasado. El precio social asume varias formas como, por ejemplo, la disminución en la calidad de vida de las ciudades, mayores gastos con salud y seguros y pérdida de la capacidad productiva de la población. El control de la contaminación ambiental es un proceso históricamente reciente. No está todavía totalmente maduro. Incluye dos tipos de costos: el costo de las instalaciones y el de la reglamentación. El primer tipo proviene de los controles de las emisiones junto a su propia fuente, generalmente en las fábricas e industrias. Son internalizados por el sector privado, generalmente por el propio contaminador, y comprenden los gastos con filtros, equipamientos 
técnicos especializados, investigaciones y perdidas relativas de potencia y energía, entre otros. Los costos de reglamentación, a su vez, son absorbidos por el sector público y comprende todos los recursos necesarios para desarrollar y hacer cumplir las normas sobre los límites máximos de contaminación, recursos con personal y equipamientos especializados. Además de las pérdidas en las actividades productivas, en la ecología y en los bienes materiales, la contaminación ambiental tiene consecuencias más serias en la salud y en el bienestar de la población. La emisión de humo, gases y demás residuos contaminantes causan enfermedades respiratorias y de otros tipos, además de otras enfermedades y muchas lesiones, con efectos temporarios o permanentes en la capacidad productiva de los individuos y en los gastos con salud y prevención de molestias. La gran cuestión, en toda la evaluación de proyectos, es cómo cuantificar el valor de las pérdidas de salubridad y de vidas humanas en determinados tipos de propuestas productivas. La ética y la moral se sustentan en la premisa social del orden necesario a través del equilibrio normativo de la sociedad y en la premisa metafísica de que el valor de la vida es absoluto y, por eso, cualquier curso de acción proyecto que causase, directa o indirectamente, el sacrificio de una única vida debería ser abominado. Sin embargo, nuevas fábricas son implantadas con eliminación de residuos, que, eventualmente, causarían enfermedades, invalidez, lesiones y pérdidas de vidas. El sistema parece tener su lógica en un índice bajo de mortalidad. Un enfoque materialista para el cálculo del valor económico de la vida, de la invalidez y de la pérdida de la salud de un individuo procura identificar como los demás individuos - y no apenas sus parientes y amigos próximos - son afectados. Con este razonamiento, conocido como del valor "presente líquido sacrificado", cada individuo se corresponde a un "proyecto" con duración limitada, que en los períodos iniciales (infancia y adolescencia) exige inversiones por parte de la sociedad (alimentación, educación, vivienda, etc.) para, en los períodos siguientes, proveer beneficios líquidos (valor de su producción menos todos los gastos de mantenimiento bajo la forma de alimentación, asistencia médica, etc.). Se trata de un abordaje radical de racionalismo económico, que "sacrifica" la vida humana, pariente próximo el concepto de "capital humano", para agrupar la estrategia educacional. Merece ser mencionado, con esta restricción, porque muchos lo aceptan como fase de razonamiento de evaluación. Los llamados bienes de mercado son aquéllos destinados al consumo, a través del mecanismo de los precios. Se orientan para satisfacer necesidades sociales o creadas por la propaganda, sin hacer del consumidor destino final. Su producción puede implicar un sacrificio para los demás individuos. A medida que todos estos bienes y servicios provean utilidad bien definida para los consumidores, el valor de esta utilidad tendrá como referencia objetivo, los precios de mercado. La categoría propuesta corresponde a los llamados "bienes públicos o colectivos". Tales bienes, o servicios, no son divisibles, en el sentido de que el consumo de un individuo no perjudica las posibilidades de consumo de los demás. El hecho de no ser eliminado, por el acto de consumo, trae algunas dificultades la medición de los beneficios sociales inherentes al consumo de estos bienes. Como no es posible medir la cantidad consumida por cada individuo, cada consumidor tiende a subestimar naturalmente los beneficios, de tal modo que, si existiese un mercado, su precio no sería muy diferente de cero. Si las preferencias no son expresadas de forma visible, no podemos cuantificar directamente los precios y, por lo tanto, no disponemos de este importante parámetro para el cálculo de los beneficios. El sistema de mercado 
no puede ser usado para evaluar beneficios que no son vendidos. En cuanto a la oferta de los bienes de mercado, es financiada por el propio ingreso con la venta de estos bienes al precio de mercado y son los estímulos de estímulo de mercado que van a determinar el flujo de producción; por otro lado, como los bienes públicos no consiguen un precio muy diferente de cero, la generación de ingresos a través del sistema de mercado es insuficiente para estimular al sector privado a producir. Por esta razón, cabe al sector público producir directamente o financiar la producción de estos bienes para la colectividad. La mayoría de las veces, los bienes públicos no son de consumo voluntario, los beneficios pueden ser usufructuados independientemente de quien ha financiado su producción. Los bienes "semipúblicos", que reúnen calidad de otros dos tipos, son divisibles de la misma forma que los bienes de mercado, pero su producción consumo tiene efectos de los más intensos en toda sociedad. Destacamos el ejemplo típico de la educación, donde el consumo directo (alumno) es perfectamente identificado; así es posible cuantificar, vía precio, parte de los beneficios de su mayor educación. Bajo este aspecto, la educación podría ser operada como el sistema de mercado. Sin embargo, la mejora del nivel de educación produce beneficios amplios y diversos para toda la sociedad y el beneficio global es muy superior a la sumatoria de los beneficios individuales. Por lo tanto, a través de esta línea de razonamiento, el sujeto central del desarrollo no es la mercadería, el mercado, el capital, el sector privado o el Estado, sino el ser humano y los demás seres vivos en sus múltiples dimensiones. Según la directiva de la declaración de la ONU, todos y cada uno de los ciudadanos son convocados a participar del desarrollo. Cada uno es llamado a ayudar en la producción de lo suficiente y hasta de lo decente para todos. Pero, aunque sea de fundamental importancia, la aprobación crítica y creativa del conocimiento no es suficiente para que se instale una conciencia ecológica colectiva. Los hombres pueden tener conocimiento, pueden hasta ser críticos y creativos, pero, si son egoístas, colocarán sus conocimientos y usarán sus capacidades apenas para atender a su individualismo posesivo - como usar sus conocimientos para tener más (individualismo-oportunismo) -, pues tener más (propiedad) significa tener más fuerza y poder que los otros (competición) y poder satisfacer todos sus deseos (consumismo). Estos son valores de la sociedad capitalista. Ésta es la lógica del capitalismo. Al contrario, se debería pautar en los valores de la sociedad, de la cooperación, del respeto, del compromiso con el prójimo, de la participación, de la responsabilidad social. Si la participación es estimulada, las personas van desarrollando su capacidad de criticar y fundamentar su crítica, deseleccionar y asumir la responsabilidad de su selección, de respetar y hacerse respetar, de comprender la fragilidad de las acciones individuales y la fuerza, de crear soluciones para los impasses con que se enfrentan, de disciplinas y organizarse colectivamente para alcanzar lo pretendido, de evaluar sus propias posibilidades y sus propios límites, de conquistar autonomía y de comprometerse cada vez más con la sociedad global, de percibirse como parte de una totalidad natural y social, cuya preservación depende de todos y de cada uno. Según Sherrill (1993), "El análisis económico, descuenta beneficios y costos futuros más que los presentes, pues la sociedad tiende a dar más valor a los beneficios presentes que a los futuros y a preferir que los costos sean pagados mañana más que hoy. Esto de modo general". Esclarece que en relación con el medio y a los recursos biológicos, esta manera de pensar es errada. Prosigue: "la tasa de descuento da valor al capital en términos de su costo de oportunidad de hoy, es 
un referencial de mercado que no se aplica a decisiones de largo plazo, como es el caso de inversión de recursos biológicos, y ni debe orientar decisiones de naturaleza cualitativa. El mercado no trabaja con el valor real, apenas con fuerzas de oferta y demanda". Los recursos naturales, normalmente, no son evaluados como capital natural y no figuran en la contabilidad nacional. En la evaluación del PIB, la renta basada en la utilización de recursos naturales no es incorporada, como tampoco son consideradas las pérdidas en el stock de recursos generados por esa renta. Si algún día, en la evaluación del PIB, imputasen como pérdida las áreas deforestadas, los incentivos a la conservación serán vistos de otra forma.

\section{REFERENCIAS}

ALMEIDA, J. R.; SILVA, C. E.; SILVA, C. V. V.; AGUIAR, L. A.; GARCIA, V. S.; SOUZA, C. P.; LENZ, E. R. S.; LINS, G. A.; ALMEIDA, S. M.. Multifatorialidade em saúde ambiental. Environmental Scientiae, v.1, p.26-47, 2019. DOI: https://doi.org/10.6008/CBPC2674-6492.2019.002.0002

ALMEIDA, J.R.; SILVA, C. E.; SILVA, C. V. V.; AGUIAR, L. A.; GARCIA, V. S.; SOUZA, C. P.; LENZ, E. R. S.; LINS, G. A.; ALMEIDA, S. M.. Política e economia de vigilância em saúde ambiental. Environmental Scientiae, v.1, p.1-25, 2019. DOI: https://doi.org/10.6008/CBPC2674-6492.2019.002.0001

ALMEIDA, J. R.; SILVA, C. E.; RODRIGUES, M. G.. Evaluation of the environmental impacts caused by deforestation in the hydric regimen of the metropolitan region of Petrópolis (RJ), Brazil. Engineering Sciences, v.1, p.14-21, 2013. DOI: https://doi.org/10.6008/ESS2318-3055.2013.001.0002

SILVA, B. M. P.; CAVALCANTI, P. P. M. S.; RODRIGUES, M. G.; ALMEIDA, J. R.. Análise do processo de licenciamento ambiental no estado do Rio De Janeiro. Revista Internacional de Ciências, v.4, p.83-106, 2014. DOI: https://doi.org/10.12957/ric.2014.13832

SILVA, C. E.; SOUZA, F. M. N.; AGUIAR, L. A.; ALMEIDA, J. R.. Análise de riscos como instrumento para sistemas de gestão ambiental. Revista Ibero-Americana de Ciências Ambientais, v.3, p.17-41, 2012. DOI:

https://doi.org/10.6008/ESS2179-6858.2012.001.0002

SILVA, L. C. L. A.; SILVA, C. E.; ALMEIDA, J. R.. Análise da implementação de um programa de sustentabilidade corporativa no Inmetro. Revista Ibero-Americana de Ciências Ambientais, v.2, p.45-58, 2011. DOI: https://doi.org/10.6008/ESS2179-6858.2011.001.0004

SOUZA, F. M. N.; SILVA, C. E.; AGUIAR, L. A.; ALMEIDA, J. R.. Proposta para utilização da simulação computacional em análise de risco, avaliação de desempenho e sistemas de gestão ambiental. Revista Ibero-Americana de Ciências Ambientais, v.2, p.39-63, 2011. DOI: https://doi.org/10.6008/ESS2179-6858.2011.002.0003

A CBPC - Companhia Brasileira de Produção Científica (CNPJ: 11.221.422/0001-03) detém os direitos materiais desta publicação. Os direitos referem-se à publicação do trabalho em qualquer parte do mundo, incluindo os direitos às renovações, expansões e disseminações da contribuição, bem como outros direitos subsidiários. Todos os trabalhos publicados eletronicamente poderão posteriormente ser publicados em coletâneas impressas sob coordenação da Sapientiae Publishing, da Companhia Brasileira de Produção Científica e seus parceiros autorizados. Os (as) autores (as) preservam os direitos autorais, mas não têm permissão para a publicação da contribuição em outro meio, impresso ou digital, em português ou em tradução. 